\title{
Stability analysis of imitative dynamics in two-community with multiple delays
}

Wenjun $\mathrm{Hu}^{1,2}$ [D and Gang Zhang ${ }^{2 *}$

\section{"Correspondence:}

gangzhang@hebtu.edu.cn

${ }^{2} S c h o o l$ of Mathematical Sciences,

Hebei Normal University,

Shijiazhuang, P.R. China

Full list of author information is

available at the end of the article

\section{Springer}

\begin{abstract}
Evolutionary game dynamics has become a hot topic in recent years. In this paper, we combine game theory and dynamical systems to investigate the stability of imitative dynamics in two-community with different kinds of delays. First, we establish a new model of imitative dynamics in two-community. Then, three sufficient conditions of the asymptotical stability for the neutrally stable strategy of the imitative dynamics are obtained. Finally, numerical simulations are consistent with the theoretical analysis.
\end{abstract}

Keywords: Imitative dynamics; Neutrally stable strategy; Asymptotical stability; Delay

\section{Introduction}

Evolutionary game dynamics propose a basic framework to study and predict the evolutionary behavior in physics [1, 2], biology [3-5], economics [6, 7], and social networks $[8,9]$. The most important approach focusing on the evolutionary game dynamics is replicator dynamic, which was first defined for single species by Taylor and Jonker [10], and then has been widely researched by many scholars [11-14]. In fact, other evolutionary dynamics have also been researched. For example, imitative dynamics [15, 16], Brown-von Neumann-Nash dynamics [17], logit dynamics [18, 19], pairwise comparison dynamics [20], etc.

Imitative dynamic is a generalization of replicator dynamic, which can spread the successful strategy more likely than replicator dynamic. In recent years, imitative dynamic has been discussed in different fields by many researchers [21-25]. Cheung [21] studied the imitative dynamics for games with continuous strategy space, they obtained global convergence and local stability results for imitative dynamics. Wang et al. [22] investigated the imitation dynamics with delay, they obtained a sufficient condition for two-phenotype imitative model and gave some numerical simulations for three-phenotype imitative model. Hu et al. [25] researched the imitative dynamics with discrete delay, they obtained some sufficient conditions of stability in imitative dynamics.

Time delay can represent those processes requiring time to occur such as incubation and transportation, which is ubiquitous in physics, biology, or social systems. Since the important effect of the time delay, many researchers have studied the replicator dynamics with

C) The Author(s) 2020. This article is licensed under a Creative Commons Attribution 4.0 International License, which permits use, sharing, adaptation, distribution and reproduction in any medium or format, as long as you give appropriate credit to the original author(s) and the source, provide a link to the Creative Commons licence, and indicate if changes were made. The images or other third party material in this article are included in the article's Creative Commons licence, unless indicated otherwise in a credit line to the material. If material is not included in the article's Creative Commons licence and your intended use is not permitted by statutory regulation or exceeds the permitted use, you will need to obtain permission directly from the copyright holder. To view a copy of this licence, visit http://creativecommons.org/licenses/by/4.0/. 
time delay [26-30]. Tao et al. [26] first proposed the effect of time delay and evolutionary stable strategy, they found that time delay would affect the stability of the replicator dynamics. Nesrine et al. [27] researched the discrete and continuous delays in replicator dynamics, they derived some necessary and sufficient delay-independent conditions for replicator dynamics. Wesson et al. [28] discussed the Hopf bifurcation in two-strategy and three-strategy delayed replicator dynamics, they showed that the bifurcation and limit cycles would exhibit in delayed replicator dynamics.

As we all know, the research in this field is mainly focused on the same community where the individual has no choice to interact with the opponent in the other communities. Nevertheless, in social networks and biology [31,32], the population is formed by different communities which can be called clusters. In this case, the individual may interact with an opponent inside or outside the community. Nesrune et al. [32] considered evolutionary dynamics in interacting communities, they researched the two-community two-strategy model and obtained some sufficient conditions for stability. However, the delay between two communities is the same, it may be different in two communities in an actual system.

Based on previous literature, we aim to generalize the model in [32] and discuss (i) the delays are diverse in two communities; (ii) a new signification parameter $\alpha(0<\alpha<1)$. In our model, the dynamics can be considered as a stochastic combination with diverse delays. We research the effect of diversity delay on the stability in a new model and obtain three sufficient conditions for stability in imitative dynamics with different delays. Our results show that the interior equilibrium in imitative dynamics is asymptotically stable when the delay is smaller than the critical value. The present paper is structured as follows. First, we recall the main concepts of evolutionary dynamics, imitative dynamics, and asymmetric delay in Sect. 2 . Then the model and stability analysis of the neutrally stable strategy is researched in Sect. 3. At last, the numerical analysis is given by snowdrift games in Sect. 4.

\section{Preliminary}

In this section, we recall neutrally stable strategy, two-community imitative dynamics, and asymmetric delay.

\subsection{Neutrally stable strategy}

In the previous literature, we have seen that the evolutionarily stable strategy (ESS) [33] is important in the evolutionary dynamics. In this paper, a special kind of ESS called neutrally stable strategy (NSS) is discussed.

Definition 2.1 ([34]) A strategy $x \in \Delta$ is an NSS if, for every strategy $y \in \Delta$, there exists some $\bar{\varepsilon}_{y} \in(0,1)$ such that the inequality

$$
u[x, \varepsilon y+(1-\varepsilon) x] \geq u[y, \varepsilon y+(1-\varepsilon) x]
$$

holds for all $\varepsilon \in\left(0, \bar{\varepsilon}_{y}\right)$, where $\Delta$ denotes the mixed-strategy set, $u[x, y]$ is the payoff when strategy $x$ interacts with strategy $y$.

The definition means that NSS is different from the ESS, while the former requires that no mutant thrive in the sense of earning a higher payoff than the incumbent strategy, the latter requires that no mutant strategy persist in the sense of earning an equal or higher payoff. 


\subsection{Two-community imitative dynamics}

The imitative dynamics can describe the evolution of various strategies in the communities. In two-community, the individual from each community may interact with an opponent from the same or different communities. Assume that $p_{i}$ denotes the probability that the opponent is from the same community $i(i=1,2)$, and $\left\{M_{i}, N_{i}\right\}$ is the strategy set in each community. The population profile can be defined by $\mathrm{x}=\left(x_{i}, y_{i}\right)$, where $x_{i}$ is the frequency of the strategy $M_{i}$ in community $i$ and $y_{i}$ is the frequency of the strategy $N_{i}$ in community $i$. The payoff matrix of the two-strategy in community is

$$
\begin{aligned}
& \begin{array}{llll}
M_{1} & N_{1} & M_{2} & N_{2}
\end{array} \\
& J=\begin{array}{l}
M_{1} \\
N_{1} \\
M_{2} \\
N_{2}
\end{array}\left(\begin{array}{llll}
a_{11} & b_{11} & a_{12} & b_{12} \\
c_{11} & d_{11} & c_{12} & d_{12} \\
a_{21} & b_{21} & a_{22} & b_{22} \\
c_{21} & d_{21} & c_{22} & d_{22}
\end{array}\right) .
\end{aligned}
$$

From the payoff matrix, one can obtain the payoff of each strategy as follows:

$$
\begin{array}{ll}
f_{M_{1}}=a_{11} x_{1}+b_{11} y_{1}+a_{12} x_{2}+b_{12} y_{2}, & f_{N_{1}}=c_{11} x_{1}+d_{11} y_{1}+c_{12} x_{2}+d_{12} y_{2}, \\
f_{M_{2}}=a_{21} x_{1}+b_{21} y_{1}+a_{22} x_{2}+b_{22} y_{2}, & f_{N_{2}}=c_{21} x_{1}+d_{21} y_{1}+c_{22} x_{2}+d_{22} y_{2} .
\end{array}
$$

The imitative dynamics in two-community can been written as follows:

$$
\left\{\begin{array}{l}
\frac{\dot{x}_{1}(t)}{x_{1}(t)}=\left[p_{1} y_{1}(t)\left(f_{N_{1}}-f_{M_{1}}\right)_{+}+\left(1-p_{1}\right)\left(x_{2}(t)\left(f_{M_{2}}-f_{M_{1}}\right)_{+}+y_{2}(t)\left(f_{N_{2}}-f_{M_{1}}\right)_{+}\right)\right], \\
\frac{\dot{y}_{1}(t)}{y_{1}(t)}=\left[p_{1} x_{1}(t)\left(f_{M_{1}}-f_{N_{1}}\right)_{+}+\left(1-p_{1}\right)\left(x_{2}(t)\left(f_{M_{2}}-f_{N_{1}}\right)_{+}+y_{2}(t)\left(f_{N_{2}}-f_{N_{1}}\right)_{+}\right)\right], \\
\frac{\dot{x}_{2}(t)}{x_{2}(t)}=\left[p_{2} y_{2}(t)\left(f_{N_{2}}-f_{M_{2}}\right)_{+}+\left(1-p_{1}\right)\left(x_{1}(t)\left(f_{M_{1}}-f_{M_{2}}\right)_{+}+y_{1}(t)\left(f_{N_{1}}-f_{M_{2}}\right)_{+}\right)\right], \\
\frac{\dot{y}_{2}(t)}{y_{2}(t)}=\left[p_{2} x_{2}(t)\left(f_{N_{1}}-f_{N_{2}}\right)_{+}+\left(1-p_{2}\right)\left(x_{1}(t)\left(f_{M_{1}}-f_{N_{2}}\right)_{+}+y_{1}(t)\left(f_{N_{1}}-f_{N_{2}}\right)_{+}\right)\right],
\end{array}\right.
$$

where $f(x)_{+}=f(x)$ if $f(x) \geq 0$ and $f(x)=0$ if $f(x)<0$.

Since $x_{1}(t)+y_{1}(t)=1, x_{2}(t)+y_{2}(t)=1$, Eq. (2.1) can be calculated and simplified as follows:

$$
\left\{\begin{array}{l}
\frac{\dot{x}_{1}(t)}{x_{1}(t)}=\left(1-x_{1}(t)\right)\left[p_{1} A_{11} x_{1}(t)+\left(1-p_{1}\right) A_{12} x_{2}(t)+C_{1}\right], \\
\frac{\dot{x}_{2}(t)}{x_{2}(t)}=\left(1-x_{2}(t)\right)\left[p_{2} A_{22} x_{2}(t)+\left(1-p_{2}\right) A_{21} x_{1}(t)+C_{2}\right],
\end{array}\right.
$$

where the parameters are presented in Table 1.

Table 1 Parameters in the model

\begin{tabular}{ll}
\hline Par. & Value \\
\hline$A_{11}$ & $a_{11}-b_{11}-c_{11}+d_{11}$ \\
$A_{22}$ & $a_{22}-b_{22}-c_{22}+d_{22}$ \\
$A_{12}$ & $a_{12}-b_{12}-c_{12}+d_{12}$ \\
$A_{21}$ & $a_{21}-b_{21}-c_{21}+d_{21}$ \\
$C_{1}$ & $p_{1}\left(b_{11}-d_{11}\right)+\left(1-p_{1}\right)\left(b_{12}-d_{12}\right)$ \\
$C_{2}$ & $p_{2}\left(b_{22}-d_{22}\right)+\left(1-p_{2}\right)\left(b_{21}-d_{21}\right)$ \\
\hline
\end{tabular}


Eq. (2.1) shows the existence of nine equilibrium points

$$
\begin{aligned}
& \left\{(0,0),(1,1),(0,1),(1,0),\left(0,-\frac{C_{2}}{p_{2} A_{22}}\right),\left(-\frac{C_{2}}{p_{2} A_{22}}, 0\right),\right. \\
& \left.\quad\left(1,-\frac{\left(1-p_{2}\right) A_{12}+C_{2}}{p_{2} A_{22}}\right),\left(-\frac{\left(1-p_{1}\right) A_{12}+C_{1}}{p_{1} A_{11}}, 1\right),\left(\frac{Q_{1}}{Q}, \frac{Q_{2}}{Q}\right)\right\},
\end{aligned}
$$

where

$$
\begin{aligned}
& Q=p_{1} p_{2} A_{11} A_{22}-\left(1-p_{1}\right)\left(1-p_{2}\right) A_{12} A_{21}, \\
& Q_{1}=\left(1-p_{1}\right) A_{12} C_{2}-p_{2} A_{22} C_{1}, \quad Q_{2}=\left(1-p_{2}\right) A_{21} C_{1}-p_{1} A_{11} C_{2} .
\end{aligned}
$$

We know that only one point is an interior equilibrium point, and denote the equilibrium as $x^{*}=\left(x_{1}^{*}, x_{2}^{*}\right)$. Refer to [32], one can obtain the stability of $E q$. (2.2).

Lemma 1 ([32]) (i) The interior equilibrium point $\mathbf{x}^{*}$ in dynamics (2.2) is asymptotically stable if $A_{11}<0, A_{22}<0$, and $Q>0$. (ii) The fully mixed NSS is asymptotically stable.

\subsection{Asymmetric delay}

Assume that $\mathrm{x}=\left(x_{1}, x_{2}\right)$ is the population profile, and $\overline{\mathrm{x}} \equiv\left(\bar{x}_{1}, \bar{x}_{2}\right)$ is the symmetric delay, where $\bar{x}_{i} \equiv x_{i}(t-\tau)$. The asymmetric delay is the case where only interactions between individuals with different strategies are delayed, while interactions between individuals with the same strategy may be instantaneous.

Therefore, we define

$$
\overline{\mathrm{x}}^{1} \equiv\left(x_{1}, \bar{x}_{2}\right), \quad \overline{\mathrm{x}}^{2} \equiv\left(\bar{x}_{1}, x_{2}\right)
$$

For ease of notation, we would introduce an incremental formula between two strategies:

$$
\overline{\mathrm{x}}_{\alpha} \equiv \overline{\mathrm{x}}+\alpha\left(\overline{\mathrm{x}}^{i}-\overline{\mathrm{x}}\right), \quad \alpha \in[0,1]
$$

Especially, (i) $\alpha=0$ means the increment is zero, i.e., the fixed delay, which has been researched in [30]; (ii) $\alpha=1$ means the case of asymmetric delay; (iii) when values of $\alpha$ are between 0 and 1 , the dynamics can be regarded as a stochastic combination. While interactions with individuals adopting the other strategy are always delayed by $\tau$, interactions with individuals adopting one's own strategy are instantaneous with probability $\alpha$ and delayed with probability $1-\alpha$.

\section{The main result}

In this section, we consider two-community imitative dynamics with diverse delays.

\subsection{Two-community with asymmetric delay}

In this subsection, we assume that the strategies take an asymmetric delay $\tau$. The corresponding imitative dynamics for the two-community is given as follows:

$$
\left\{\begin{array}{l}
\frac{\dot{x}_{1}(t)}{x_{1}(t)}=\left(1-x_{1}(t)\right)\left[p_{1} A_{11}\left(\alpha x_{1}(t)+(1-\alpha) x_{1}(t-\tau)\right)+\left(1-p_{1}\right) A_{12} x_{2}(t-\tau)+C_{1}\right], \\
\frac{\dot{x}_{2}(t)}{x_{2}(t)}=\left(1-x_{2}(t)\right)\left[p_{2} A_{22}\left(\alpha\left(x_{1}(t)+(1-\alpha) x_{2}(t-\tau)\right)+\left(1-p_{2}\right) A_{21} x_{1}(t-\tau)+C_{2}\right],\right.
\end{array}\right.
$$

where $A_{11}, A_{22}, A_{12}, A_{21}, C_{1}, C_{2}$ are given in Table 1. 
For convenience of research, we introduce a small perturbation around $\mathbf{x}^{*}: z_{1}(t)=x_{1}(t)-$ $x_{1}^{*}, z_{2}(t)=x_{2}(t)-x_{2}^{*}$. Then we can obtain the linearized form of $E q$. (3.1):

$$
\left\{\begin{array}{l}
\dot{z_{1}}(t)=\delta_{1}\left[p_{1} A_{11}\left(\alpha z_{1}(t)+(1-\alpha) z_{1}(t-\tau)\right)+\left(1-p_{1}\right) A_{12} z_{2}(t-\tau)\right] \\
\dot{z}_{2}(t)=\delta_{2}\left[p_{2} A_{22}\left(\alpha z_{2}(t)+(1-\alpha) z_{2}(t-\tau)\right)+\left(1-p_{2}\right) A_{21} z_{1}(t-\tau)\right]
\end{array}\right.
$$

where $\delta_{1}=x_{1}^{*}\left(1-x_{1}^{*}\right)$ and $\delta_{2}=x_{2}^{*}\left(1-x_{2}^{*}\right)$. Linear Eq. (3.2) is of the form

$$
\dot{x}(t)=A x(t-\tau),
$$

which has been discussed in [35]. Similar to the results in the literature, we obtain the theorem for the stability of the mixed NSS for Eq. (3.1).

Theorem 1 The mixed NSS $\boldsymbol{x}^{*}$ in Eq. (3.1) is asymptotically stable if

$$
\tau<\bar{\tau}=\min \left\{\frac{1}{k_{1}(1-\alpha)}, \frac{k_{1}}{2\left(k_{2}(1-\alpha)+k_{3}\right)}\right\},
$$

where

$$
\begin{aligned}
& k_{1}=-\left(\delta_{1} p_{1} A_{11}+\delta_{2} p_{2} A_{22}\right), \quad k_{2}=\delta_{1} \delta_{2} p_{1} p_{2} A_{11} A_{22}, \\
& k_{3}=-\delta_{1} \delta_{2}\left(1-p_{1}\right)\left(1-p_{2}\right) A_{12} A_{21} .
\end{aligned}
$$

Proof Taking the Laplace transform of system (3.2), we can obtain the corresponding characteristic equation as follows:

$$
\begin{aligned}
& \lambda^{2}-\left(\delta_{1} p_{1} A_{11}+\delta_{2} p_{2} A_{22}\right)\left(e^{-\lambda \tau}+\alpha\left(1-e^{-\lambda \tau}\right)\right) \lambda \\
& \quad+\delta_{1} \delta_{2}\left[p_{1} p_{2} A_{11} A_{22}\left(e^{-\lambda \tau}+\alpha\left(1-e^{-\lambda \tau}\right)\right)^{2}-\left(1-p_{1}\right)\left(1-p_{2}\right) A_{12} A_{21} e^{-2 \lambda \tau}\right]=0 .
\end{aligned}
$$

By substituting the exponential term with a Taylor series expansion and keeping only linear terms in $\tau$ in the above equation, one can obtain the simplified characteristic equation

$$
\left(1-k_{1}(1-\alpha) \tau\right) \lambda^{2}+\left(k_{1}+2 k_{3} \tau-2 k_{2}(1-\alpha) \tau\right) \lambda+k_{2}+k_{3}=0
$$

The zero solution is asymptotically stable if all solutions of (3.2) have negative real parts, i.e., $\mathbf{x}^{*}$ is asymptotically stable if all the roots of the characteristic equation have negative real parts. In this case, the roots of Eq. (3.2) have negative real parts if their product is positive and their sum is negative:

$$
\left\{\begin{array}{l}
\frac{k_{2}+k_{3}}{1-k_{1}(1-\alpha) \tau}>0 \\
-\frac{k_{1}-2 k_{2}(1-\alpha) \tau-2 k_{3} \tau}{1-k_{1}(1-\alpha) \tau}<0 .
\end{array}\right.
$$

By Lemma 1, one can obtain $k_{1}>0, k_{2}+k_{3}=\delta_{1} \delta_{2} Q>0$. Therefore, the condition (i) yields $\tau<\frac{1}{k_{1}(1-\alpha)}$. Condition (ii) yields:

$$
\begin{aligned}
& \text { if }\left(k_{2}(1-\alpha)-k_{3}\right)>0 \text {, then } \tau<\frac{k_{1}}{2\left(k_{2}(1-\alpha)+k_{3}\right)} ; \\
& \text { if }\left(k_{2}(1-\alpha)+k_{3}\right)<0 \text {, the conclusion would be established for all } \tau \text {. }
\end{aligned}
$$


In a sum, we can obtain $\tau<\bar{\tau}=\min \left\{\frac{1}{k_{1}(1-\alpha)}, \frac{k_{1}}{2\left(k_{2}(1-\alpha)+k_{3}\right)}\right\}$.

Remark 3.1 The result in Theorem 1 gives an upper bound on strategic delays for which NSS remains asymptotically stable in two-community with asymmetric delay.

\subsection{Two-community with asymmetric and diverse delays}

In this subsection, we research two kinds of different delays in two-community.

\subsubsection{Two-community with asymmetric delay}

Assume that $\tau_{1}$ denotes the delay associated with the inter-community interactions and $\tau_{2}$ denotes that two individuals from different communities get involved in an interaction.

The corresponding imitative dynamics for the two-community is given by

$$
\left\{\begin{array}{l}
\frac{\dot{x}_{1}(t)}{x_{1}(t)}=\left(1-x_{1}(t)\right)\left[p_{1} A_{11}\left(\alpha x_{1}(t)+(1-\alpha) x_{1}\left(t-\tau_{1}\right)\right)+\left(1-p_{1}\right) A_{12} x_{2}\left(t-\tau_{2}\right)+C_{1}\right] \\
\frac{\dot{x}_{2}(t)}{x_{2}(t)}=\left(1-x_{2}(t)\right)\left[p_{2} A_{22}\left(\alpha x_{2}(t)+(1-\alpha) x_{2}\left(t-\tau_{1}\right)\right)+\left(1-p_{2}\right) A_{21} x_{1}\left(t-\tau_{2}\right)+C_{2}\right]
\end{array}\right.
$$

where $A_{11}, A_{22}, A_{12}, A_{21}, C_{1}, C_{2}$ are given in Sect. 2.2. Using the similar linearization in the above subsection, we can obtain the linearized form of $E q$. (3.4) as follows:

$$
\left\{\begin{array}{l}
\dot{z}_{1}(t)=\delta_{1}\left[p_{1} A_{11}\left(\alpha z_{1}(t)+(1-\alpha) z_{1}(t-\tau)\right)+\left(1-p_{1}\right) A_{12} z_{2}\left(t-\tau_{2}\right)\right] \\
\dot{z_{2}}(t)=\delta_{2}\left[p_{2} A_{22}\left(\alpha z_{2}(t)+(1-\alpha) z_{2}(t-\tau)\right)+\left(1-p_{2}\right) A_{21} z_{1}\left(t-\tau_{2}\right)\right]
\end{array}\right.
$$

Linear Eq. (3.5) is of the form

$$
\dot{x}(t)=A x\left(t-\tau_{1}\right)+B x\left(t-\tau_{2}\right) .
$$

Similar to the results in [35], we obtain the theorem on the stability of the NSS for Eq. (3.4).

Theorem 2 If (i) $\tau_{1}<\bar{\tau}=\frac{1}{k_{1}(1-\alpha)}$ and (ii) $k_{2}(1-\alpha) \tau_{1}+k_{3} \tau_{2}<\frac{k_{1}}{2}$, the mixed NSS $\boldsymbol{x}^{*}$ in Eq. (3.4) is asymptotically stable, where $k_{1}, k_{2}$, and $k_{3}$ are given in Theorem 1.

Proof Similar to the proof in Theorem 1, the corresponding characteristic equation can be written as follows:

$$
\left(1-k_{1}(1-\alpha) \tau_{1}\right) \lambda^{2}+\left(k_{1}+2 k_{3} \tau_{2}-2 k_{2}(1-\alpha) \tau_{1}\right) \lambda+k_{2}+k_{3}=0
$$

The roots of Eq. (3.6) have negative real parts if their product is positive and their sum is negative, i.e.,

$$
\left\{\begin{array}{l}
\frac{k_{2}+k_{3}}{1-k_{1}(1-\alpha) \tau_{1}}>0 \\
-\frac{k_{1}-2 k_{2}(1-\alpha) \tau_{1}-2 k_{3} \tau_{2}}{1-k_{1}(1-\alpha) \tau_{1}}<0
\end{array}\right.
$$

Using similar methods in the proof of Theorem 1, one can obtain $\tau_{1}<\bar{\tau}=\frac{1}{k_{1}(1-\alpha)}$ and (ii) $k_{2}(1-\alpha) \tau_{1}+k_{3} \tau_{2}<\frac{k_{1}}{2}$.

Remark 3.2 The result in Theorem 2 gives an upper bound of $\tau_{1}$ and the linear combination of $\tau_{1}$ and $\tau_{2}$ for which the mixed NSS remains asymptotically stable in two-community with different delays. 


\subsubsection{Two-community with diverse delays}

Let $\tau_{i}$ be the delay associated with the inter community $i$, and $\tau_{12}$ be the delay when two individuals from different communities get involved in an interaction.

The corresponding imitative dynamics for the two-community are given by

$$
\left\{\begin{aligned}
\frac{\dot{x}_{1}(t)}{x_{1}(t)}= & \left(1-x_{1}(t)\right)\left[p_{1} A_{11}\left(\alpha x_{1}(t)+(1-\alpha) x_{1}\left(t-\tau_{1}\right)\right)\right. \\
& \left.+C_{1}+\left(1-p_{1}\right) A_{12} x_{2}\left(t-\tau_{12}\right)\right], \\
\frac{\dot{x}_{2}(t)}{x_{2}(t)}= & \left(1-x_{2}(t)\right)\left[p_{2} A_{22}\left(\alpha x_{2}(t)+(1-\alpha) x_{2}\left(t-\tau_{2}\right)\right)\right. \\
& \left.+C_{2}+\left(1-p_{2}\right) A_{21} x_{1}\left(t-\tau_{12}\right)\right] .
\end{aligned}\right.
$$

Similarly, one can obtain the linearized form of Eq. (3.7) as follows:

$$
\left\{\begin{array}{l}
\dot{z_{1}}(t)=\delta_{1}\left[p_{1} A_{11}\left(\alpha z_{1}(t)+(1-\alpha) z_{1}\left(t-\tau_{1}\right)\right)+\left(1-p_{1}\right) A_{12} z_{2}\left(t-\tau_{12}\right)\right] \\
\dot{z_{2}}(t)=\delta_{2}\left[p_{2} A_{22}\left(\alpha z_{1}(t)+(1-\alpha) z_{1}\left(t-\tau_{2}\right)\right)+\left(1-p_{2}\right) A_{21} z_{1}\left(t-\tau_{12}\right)\right] .
\end{array}\right.
$$

Linear Eq. (3.8) is of the form

$$
\dot{x}(t)=A x\left(t-\tau_{1}\right)+B x\left(t-\tau_{2}\right)+C x\left(t-\tau_{3}\right) .
$$

Similar to the results, we obtain the theorem on the stability of the NSS for Eq. (3.7).

Theorem 3 If (i) $k_{11} \tau_{1}+k_{12} \tau_{2}<\bar{\tau}=\frac{1}{(1-\alpha)}$ and (ii) $k_{2}(1-\alpha)\left(\tau_{1}+\tau_{2}\right)+2 k_{3} \tau_{12}<k_{1}$, the mixed NSS $\boldsymbol{x}^{*}$ in Eq. (3.7) is asymptotically stable, where $k_{11}=-\delta_{1} p_{1} A_{11}$ and $k_{12}=-\delta_{2} p_{2} A_{22}$, and $k_{1}, k_{2}$, and $k_{3}$ are given in Theorem 1.

Using similar methods in the proof of Theorem 2, one can obtain (i) $k_{11} \tau_{1}+k_{12} \tau_{2}<\bar{\tau}=$ $\frac{1}{(1-\alpha)}$ and (ii) $k_{2}(1-\alpha)\left(\tau_{1}+\tau_{2}\right)+2 k_{3} \tau_{12}<k_{1}$.

Remark 3.3 The result in Theorem 3 gives an upper bound of the linear combination of $\tau_{1}, \tau_{2}$, and $\tau_{3}$ for which the mixed NSS remains asymptotically stable in two-community with different delays.

\section{Numerical simulation}

In this section, we give numerical simulation to verify that our results are effective. We consider the classical snowdrift game [36,37], where the player may use a cooperate (C) strategy or a defect (D) strategy. According to the relevant literature, we can get the payoff matrix of the snowdrift game as follows:

$$
\begin{aligned}
& \text { C D } \\
& \begin{array}{l}
C \\
D
\end{array}\left(\begin{array}{cc}
b-\frac{c}{2} & b-c \\
b & 0
\end{array}\right), \quad b>c>0,
\end{aligned}
$$

where $b$ represents the payoff of going home and $c$ represents the cost of shoveling snow. We apply our model to the snowdrift game played in a population composed of two communities of players having different behaviors. Players in community 1 are more selfless, and players in community 2 are more selfish. 
In this case, the payoff between communities 1 and 2 is described by the matrix

$$
J=\begin{array}{cccc}
C_{1} & D_{1} & C_{2} & D_{2} \\
C_{1} \\
D_{1} \\
C_{2} \\
D_{2}
\end{array}\left(\begin{array}{cccc}
b-\frac{c}{2} & b-c & b-\omega c & b-c \\
b & 0 & b & 0 \\
b-(1-\omega) c & b-c & b-\frac{c}{2} & b-c \\
b & 0 & b & 0
\end{array}\right),
$$

where $\omega(0.5<\omega<1)$ is the proposition of the cost when a selfless player competes with a selfish player.

Example 1 For the parameter values

$$
b=5, \quad c=3, \quad \omega=0.7, \quad p_{1}=0.6, \quad p_{2}=0.7,
$$

we can conclude that the mixed intermediate ESS is $s^{*}=\left(s_{1}^{*}, s_{2}^{*}\right)=(0.4357,0.6616)$. When the initial (arbitrary) is given by $(0.65,0.35)$, the maximum value of the delay for the intermediate NSS is asymptotically stable is $\bar{\tau}=2.3478$. In Fig. 1, we observe a convergence to the intermediate NSS $s^{*}$ when $\tau<\bar{\tau}$. However, the oscillation phenomenon appears when $\tau>\bar{\tau}$ (see Fig. 1). At the same time, the result implies that the amplitude and the period of the solution will increase with the increase of $\tau$ (see Fig. 2).

Example 1 explains the effect of the same delay on the stability of the NSS, which is consistent with Theorem 1.
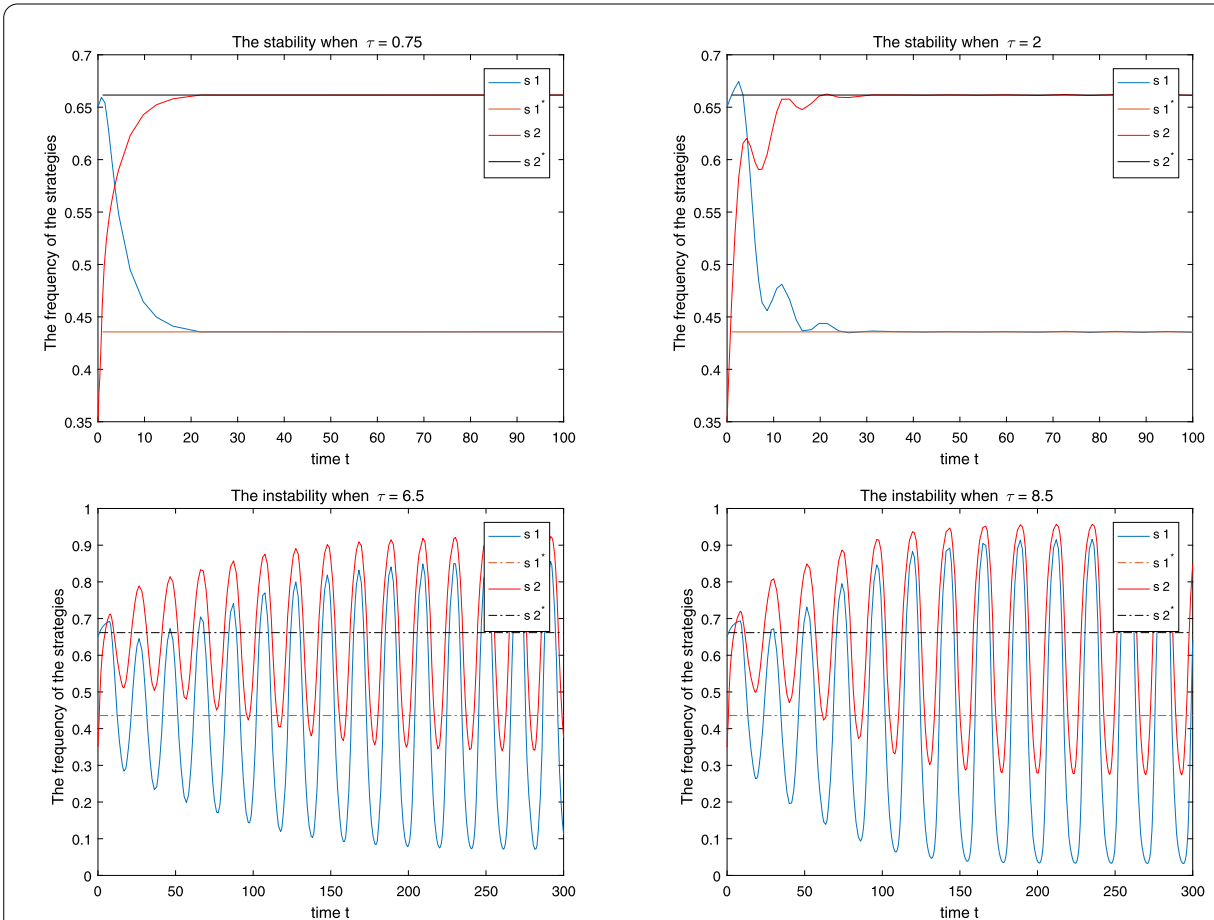

Figure 1 The stability and instability of the mixed NSS with delay 
Figure 2 The effect of $\tau$ in the replicator dynamics
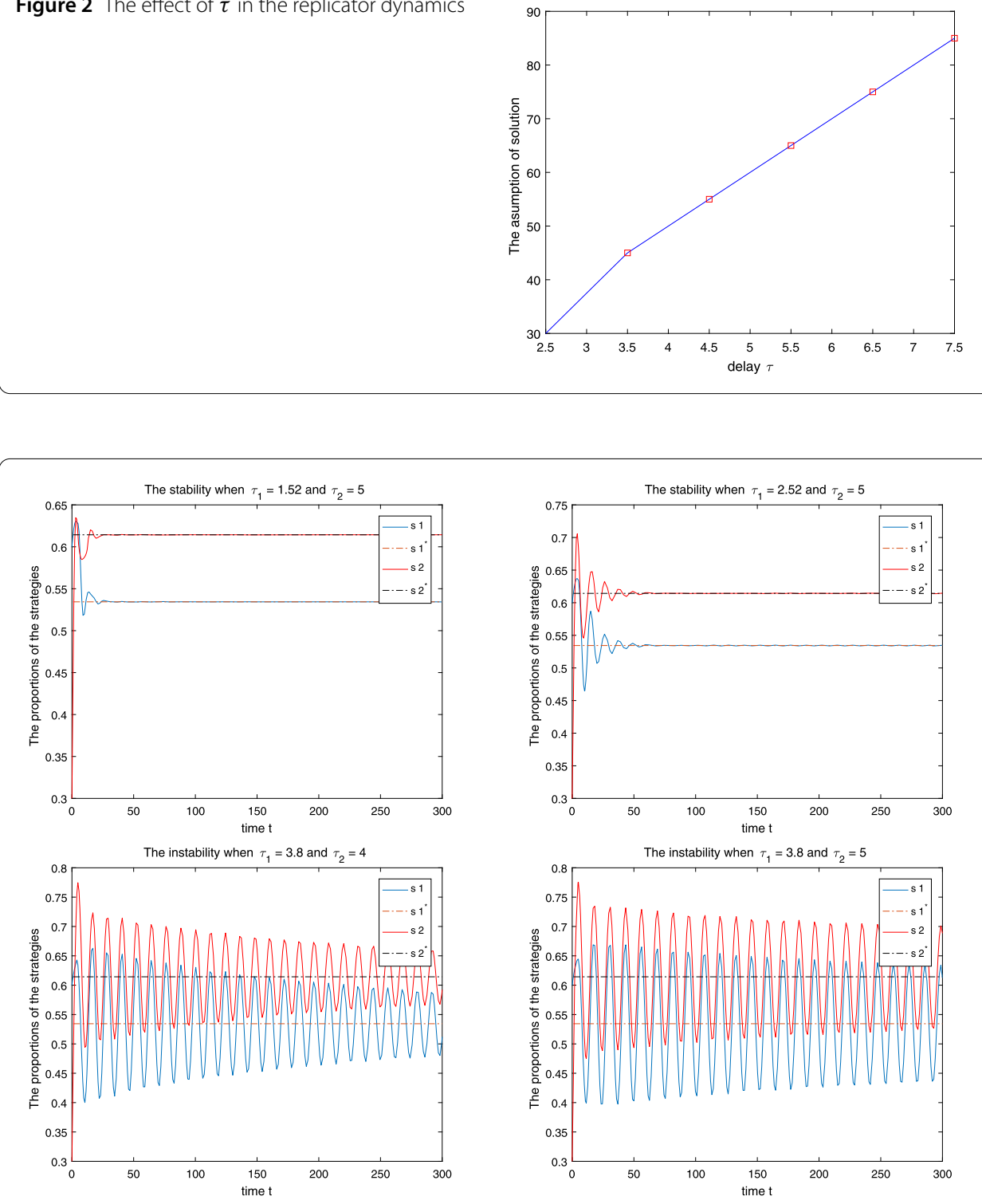

Figure 3 The stability and instability of the mixed NSS with two delays

Example 2 The parameter values are given as follows:

$$
b=5, \quad c=3, \quad \omega=0.7, \quad p_{1}=0.6, \quad p_{2}=0.7 .
$$

According to the conditions in Theorem 2, we obtain that the stability should satisfy two conditions: (i) $\tau_{1}+\tau_{2}<10.0931$ and (ii) $0.5163 \tau_{1}+0.5485 \tau_{2}<2.5$. In Fig. 3 , it is clear that the intermediate NSS is stable when $\tau_{1}$ and $\tau_{2}$ satisfy the conditions, but when the linear combination of $\tau_{1}$ and $\tau_{2}$ does not satisfy condition (i) or (ii), then $s^{*}$ is unstable.

Example 2 verifies the effect of different delays (case 1) on the stability of the mixed NSS, which is consistent with Theorem 2. 


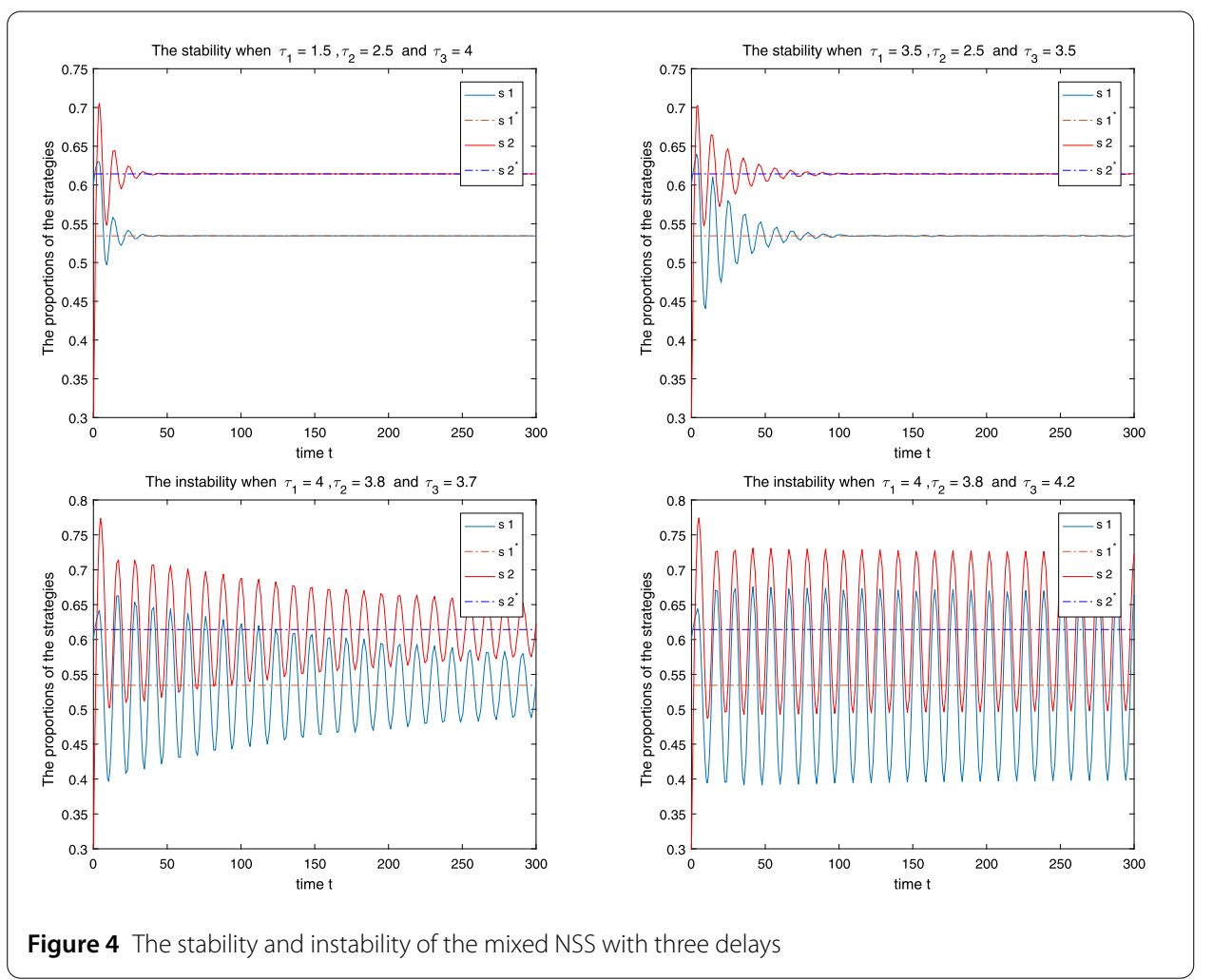

Example 3 We discuss the effect of different delays (case 2) on the stability of the intermediate ESS. The parameter values are given as follows:

$$
b=5, \quad c=3, \quad \omega=0.7, \quad p_{1}=0.8, \quad p_{2}=0.75, \quad \alpha=0.4 .
$$

Assume that (i) $0.6967 \tau_{1}+0.6219 \tau_{2}<1.6667$ and (ii) $0.26\left(\tau_{1}+\tau_{2}\right)-0.07 \tau_{12}<1.3186$. In Fig. 4, it is clear that the NSS is stable when $\tau_{1}$ and $\tau_{2}$ satisfy the conditions, and when the linear combination of $\tau_{1}$ and $\tau_{2}$ does not satisfy condition (i) or (ii), then $s^{*}$ is unstable.

Example 3 describes the effect of off-diagonal and different delay (case 2) on the stability of the mixed NSS, which is consistent with Theorem 3.

\section{Conclusion}

In this paper, the stability of the NSS in two-community has been investigated for the imitative dynamics with diverse delays. Compared with the model in [32], we introduce a new signification parameter $\alpha$ and discuss the different delays in different communities, which is more meaningful in actual social networks. Three sufficient conditions for the imitative dynamics with diverse delays are derived. The results indicate that the different delays have different effects in the community, which is different from the results in [32].

Furthermore, we will research the two-community imitative dynamics with continuous distributed delays. At the same time, the current theoretical model and numerical simulation are only considered in the imitative dynamics; we plan to discuss the stability for other dynamics such as logit dynamics or best-response dynamics. 


\section{Acknowledgements}

We are thankful to the editor and the anonymous reviewers for many valuable suggestions to improve this paper.

\section{Funding}

This paper is supported by the Scientific and Technological Innovation Programs of Higher Education Institutions in Shanxi province (No. 2019L0940) and the Natural Science Foundation of Shanxi province (No. 201901D211449).

\section{Availability of data and materials}

Data sharing not applicable to this article as no datasets were generated or analysed during the current study.

\section{Competing interests}

The authors declare that they have no competing interests.

\section{Authors' contributions}

All authors contributed equally to the manuscript and typed, read, and approved the final manuscript.

\section{Author details}

'Department of Mathematics, Luliang University, Lüliang, P.R. China. ${ }^{2}$ School of Mathematical Sciences, Hebei Normal University, Shijiazhuang, P.R. China.

\section{Publisher's Note}

Springer Nature remains neutral with regard to jurisdictional claims in published maps and institutional affiliations.

Received: 27 December 2019 Accepted: 24 May 2020 Published online: 03 June 2020

\section{References}

1. Vilone, D., Robledo, A., Sanchez, A.: Chaos and unpredictability in evolutionary dynamics in discrete time. Phys. Rev. Lett. 107, Article ID 038101 (2011)

2. Hindersin, L., Wu, B., Traulsen, A., García, J.: Computation and simulation of evolutionary game dynamics in finite populations. Sci. Rep. 9, Article ID 6946 (2019)

3. Nowak, M.A.: Five rules for the evolution of cooperation. Science 314, 1560-1563 (2006)

4. Wei, Z.C., Zhu, B., Yang, J., Perc, M., Slavinec, M.: Bifurcation analysis of two disc dynamos with viscous friction and multiple time delays. Appl. Math. Comput. 347, 265-281 (2019)

5. Lu, L.Q., Ouyang, Y.F.: Dynamic vaccination game in a heterogeneous mixing population. Physica A 533, Article ID $122032(2019)$

6. Friedman, D.: Evolutionary games in economics. Econometrica 59, 637-666 (1991)

7. Wen, J.H., Weng, P.X.: Stability and Hopf bifurcation of a producer-scrounger model with age-structure. Adv. Differ. Equ. 2016, Article ID 243 (2016)

8. Qing, P., Xiao, M., Huang, C.D., Jiang, G.P., Qiu, J.L., Lin, J.X., Wang, Z.X., Zheng, C.: Stability and bifurcation analysis of a gene expression model with small RNAs and mixed delays. Adv. Differ. Equ. 2019, Article ID 240 (2019)

9. Yang, H.X., Chen, X.J.: Promoting cooperation by punishing minority. Appl. Math. Comput. 316, 460-466 (2018)

10. Taylor, P.D., Jonker, L.B.: Evolutionarily stable strategies and game dynamics. Math. Biosci. 40, 145-156 (1978)

11. Cressman, R., Tao, Y.: The replicator equation and other game dynamics. Proc. Natl. Acad. Sci. USA 111, 10810-10817 (2014)

12. Hofbauer, J., Sigmund, K.: Evolutionary Games and Population Dynamics. Cambridge University Press, Cambridge (1998)

13. Dhiman, A., Poria, S.: Allee effect induced diversity in evolutionary dynamics. Chaos Solitons Fractals 108, 32-38 (2018)

14. Liu, J.J., Wang, J.M.: Stabilization of one-dimensional wave equation with nonlinear boundary condition subject to boundary control matched disturbance. IEEE Trans. Autom. Control 64(7), 3068-3073 (2019)

15. Hu, W.J., Tian, H.Y., Zhang, G.: Bifurcation analysis of three-strategy imitative dynamics with mutations. Complexity 2019, Article ID 4134105 (2019)

16. Hu, W.J., Zhang, G., Tian, H.Y., Wang, Z.W.: Chaotic dynamics in asymmetric rock-paper-scissors games. IEEE Access 7(1), 175614-175621 (2019)

17. Hofbauer, J., Oechssler, J., Riedel, F.: Brown-von Neumann-Nash dynamics: the continuous strategy case. Games Econ. Behav. 65, 406-429 (2009)

18. Lahkar, R., Riedel, F.: The logit dynamic for games with continuous strategy sets. Games Econ. Behav. 91, 268-282 (2015)

19. Umezuki, Y: Bifurcation analysis of the rock-paper-scissors game with discrete-time logit dynamics. Math. Soc. Sci. 95, 54-65 (2018)

20. Cheung, M.W.: Pairwise comparison dynamics for games with continuous strategy space. J. Econ. Theory 153 344-375 (2014)

21. Cheung, M.W.: Imitative dynamics for games with continuous strategy space. Games Econ. Behav. 99, 206-223 (2016)

22. Wang, S.C., Yu, J.R., Kurokawa, S., Tao, Y.: Imitation dynamics with time delay. J. Theor. Biol. 420, 8-11 (2017)

23. Baiardi, L.C., Naimzada, A.K.: Imitative and best response behaviors in a nonlinear Cournotian setting. Chaos 28 Article ID 055913 (2018)

24. Wang, X.L., Luo, C., Ding, S., Wang, J.C.: Imitating contributed players promotes cooperation in the Prisoner's Dilemma game. IEEE Access 6, 53265-53271 (2018)

25. Hu, W.J., Zhang, G., Tian, H.Y.: The stability of imitation dynamics with discrete distributed delays. Physica A 521, 218-224 (2019)

26. Tao, Y., Wang, Z:: Effect of time delay and evolutionarily stable strategy. J. Theor. Biol. 187, 111-116 (1997) 
27. Nesrine, B.K., Rachid, E.A., Yezekael, H.: Discrete and continuous distributed delays in replicator dynamics. Dyn. Games Appl. 6, 1-20 (2017)

28. Wesson, E., Rand, R.: Hopf bifurcations in delayed rock-paper-scissors replicator dynamics. Dyn. Games Appl. 6 , 139-156 (2016)

29. Yi, X., Liu, G.R.: Analysis of stochastic Nicholson-type delay system with patch structure. Appl. Math. Lett. 96, 223-229 (2019)

30. Wesson, E., Rand, R.: Hopf bifurcations in two-strategy delayed replicator dynamics. Int. J. Bifurc. Chaos 26(1), Article ID 1650006 (2016)

31. Lieberman, E., Hauert, C., Nowak, M.A.: Evolutionary dynamics on graphs. Nature 433, 312-316 (2005)

32. Nesrine, B.K., Rachid, E.A.: Evolutionary games in interacting communities. Dyn. Games Appl. 7, 131-156 (2017)

33. Smith, J.M.: The theory of games and evolution of animal conflicts. J. Theor. Biol. 47, 209-221 (1974)

34. Weibull, J.W.: Evolutionary Game Theory. MIT Press, Cambridge (1995)

35. Hara, T., Sugie J.: Stability region for systems of differential-difference equations. Funkc. Ekvacioj 39(1), 69-86 (1996)

36. Ye, W.X., Fan, S.H.: Evolutionary snowdrift game with rational selection based on radical evaluation. Appl. Math. Comput. 294, 310-317 (2017)

37. Sui, X.K., Cong, R., Li, K., Wang, L.: Evolutionary dynamics of N-person snowdrift game. Phys. Lett. A 379, 2922-2934 (2015)

\section{Submit your manuscript to a SpringerOpen ${ }^{\circ}$ journal and benefit from:}

- Convenient online submission

- Rigorous peer review

Open access: articles freely available online

- High visibility within the field

- Retaining the copyright to your article

Submit your next manuscript at $\boldsymbol{\nabla}$ springeropen.com 